\title{
Joint Hypermobility Syndrome and Postural Orthostatic Tachycardia Syndrome (HyPOTS)
}

\author{
Dana Mandel ${ }^{1 *}$, Ali D. Askari ${ }^{1}$, Charles J. Malemud ${ }^{1}$, and Artan Kaso ${ }^{1}$ \\ ${ }^{1}$ Department of Medicine, Division of Rheumatic Diseases, Case Western Reserve University School of Medicine, University Hospitals Cleveland Medical Center,
} USA

\begin{abstract}
Objective: To evaluate the association between joint hypermobility syndrome associated with postural orthostatic tachycardia syndrome (HyPOTS) and fibromyalgia.

Methods: A chart review of patients followed at two outpatient Rheumatology facilities within one hospital system were analyzed. Thirty-seven patients ( 3 male, 34 female) diagnosed with HyPOTS and fibromyalgia were reviewed.

Results: HyPOTS is a chronically disabling musculoskeletal disorder presenting clinically as widespread musculoskeletal pain and/or fatigue with joint hypermobility. However, HyPOTS may be inadvertently diagnosed as chronic fatigue syndrome or fibromyalgia. In fact, the group of fibromyalgia patients evaluated at a rheumatology outpatient clinic were found to meet the clinical criteria for HyPOTS.

Conclusions: Patients diagnosed with fibromyalgia and chronic fatigue syndrome may benefit from a further evaluation for HyPOTS. Thus, if HyPOTS is properly defined, a wide range of therapeutic options for these patients become available in order to improve their physical manifestations and quality of life.
\end{abstract}

\section{Introduction}

Often, the most common complaints voiced by patients in a rheumatology office are that of increased arthralgias, myalgias, and constitutional symptoms. It is not uncommon to receive a referral for evaluation of a patient with complaints of arthralgias, myalgias and other constitutional symptoms but without obvious signs of synovitis or connective tissue disease. It is also common to have these complaints attributed to patient's underlying disease due to their nonspecific nature. However, nonspecific arthralgias, myalgias, and fatigue are just that - nonspecific. Yet, these symptoms can lead to significant decline in a patient's quality of life. As physicians, we consistently strive to treat disease and to improve a patient's quality of life. This may mean prescribing a treatment strategy, including immunosuppressive therapy, and taking on the risk of increased side-effects. We propose that in select patients, joint hypermobility may be contributing to these non-specific complaints and that recognition and intervention can help to improve the patient's quality of life.

Joint hypermobility syndrome (JHS) is a chronic, disabling disorder which manifests as widespread musculoskeletal pain and/or fatigue in the presence of generalized joint hypermobility. Joint hypermobility can exist without any associated musculoskeletal disorder. Although a limited literature is available on joint hypermobility; approximately $5 \%$ of the adult Caucasian population has JHS with an increased prevalence noted in Asian and African-American populations [1]. Furthermore, the prevalence of JHS is noted to be approximately $10-30 \%$ in the juvenile population which decreases with age [2]. Despite the fact that there is a limited understanding of the pathophysiology of JHS, genetics do appear to play a role. For example, in studies performed on the expression of the tenascin- $X$ gene, the protein product of the gene has been noted to be present in low amounts in patients diagnosed with JHS and the Ehlers-Danlos Syndrome (hypermobility type) [2].
Furthermore, environmental triggers, such as viral illness or extended periods of deconditioning, have been proposed as causes for benign joint hypermobility transitioning into JHS [2].

In addition to musculoskeletal pain and fatigue, a subset of patients with JHS have also been found to suffer from visceral manifestations and postural tachycardia with orthostatic intolerance. Visceral manifestations often center upon gastrointestinal dysfunction. Of note, abnormalities in proprioception have also been observed in patients with hypermobility along with exaggerated blood pooling in the lower extremities, suggesting some sort of molecular defects in blood vessels [3]. Postural orthostatic tachycardia syndrome, (POTS), is also noted in patients with collagen disorders and hypermobility, which is defined as an increase in heart rate greater than 30 beats/minute within 10 minutes of standing despite a lack of decline in blood pressure. Patients often report pre-syncopal or syncopal events and associated feelings of lightheadedness, nausea and palpitations [4].

POTS has been found to be associated with sympathetic hyperactivity and small fiber neuropathy. Similarly, fibromyalgia has been associated with sympathetic hyperactivity [5]. Although there is minimal research available, case reports of adults diagnosed with fibromyalgia/chronic fatigue syndrome [2] and studies of joint

Correspondence to: Dana Mandel, Department of Medicine, Division of Rheumatic Diseases, University Hospitals Cleveland Medical Center, Foley Medical Building, 2061 Cornell Road, Room 201, Cleveland, OH 44106-5076, USA, Tel: (216) 844-2289; Fax: (216) 844-2288

Key words: HyPOTS, fibromyalgia

Received: February 17, 2017; Accepted: March 22, 2017; Published: March 25, 2017 
hypermobility performed in juveniles diagnosed with chronic fatigue syndrome (CFS) [6] shed light on patients who may inadvertently be diagnosed with fibromyalgia/CFS in the setting of joint hypermobility and POTS, or HyPOTS as we have termed this clinical entity at our medical facility.

\section{Materials and methods}

\section{Case studies and chart review}

Initially, we reviewed a case of a patient diagnosed with lupus and joint hypermobility whose clinical presentation was consistent with HyPOTS. This case review led to us to construct a protocol for review by the University Hospitals Cleveland Medical Center IRB for a review of the charts of patients with joint hypermobility and to enable us to report on clinical manifestations. This chart review study was carried out in compliance with the Helsinki Declaration. Thus, following IRB Protocol \#09-13-25, entitled, Joint Hypermobility Syndrome and POTS (HyPOTS) was approved to review the charts of patients seen at two outpatient Rheumatology facilities of University Hospitals Cleveland Medical Center. The charts of 37 patients between the ages of 18 and 58 who were diagnosed with JHS using the Brighton 1998 Criteria for the diagnosis of benign joint hypermobility syndrome were reviewed [7].

All of the patients included in this chart review study had presented with one or more of the following symptoms: chronic fatigue, generalized musculoskeletal pain (arthralgia/myalgia), autonomic dysfunctions (e.g. pre-syncopal events, palpitations, lightheadedness, hypotension), gastrointestinal symptoms (i.e. irritable bowel syndrome type), and anxiety.

Additionally, all of the patients carried the diagnostic label of fibromyalgia. Similar to research previously performed on JHS, there was a female predominance. In that regard, males made up only 3 of 37 patients who were evaluated. The mean age of these patients at the time of clinical evaluation was 34.6 years.

The charts of these 37 patients with a diagnosis of JHS were analyzed (Table 1). Twenty-three (62\%) of the patients had a positive tilt table test consistent with the diagnosis of postural orthostatic tachycardia syndrome (POTS) whereas 7 (19\%) of these patients had a negative tilt table test. Seven patients (19\%) did not have a tilt table performed at time of this analysis because of multiple reasons, including financial limitations and non-compliance.

Along with fulfilling the Brighton 1998 Criteria for JHS, the patients included in our case study were noted to have clinical signs of autonomic dysfunction, including reported pre-syncopal events and lightheadedness. After applying a standard of care treatment regimen, including education, physical therapy, increased salt and water intake, mineralocorticoid supplementation and compression stockings, a chart analysis documented improvement in the clinical symptoms of all patients analyzed.

\section{Discussion}

The case series study was performed at a Rheumatology outpatient facility after noticing a trend towards joint hypermobility in certain patients with associated reports of fatigue and fibromyalgia-type symptoms. After treating multiple patients with joint hypermobility, often in patients with primary connective tissue disease, the significant disability and decrease in reported quality of life amongst these patients with joint hypermobility was apparent. Although these patients often had adequate control of their underlying disorders they reported significant suffering and symptomatology, which was not relieved with
Abbreviations for patients with concurrent rheumatic diseases; SpA: Spondyloarthropathy; UCTD: Undifferentiated Connective Tissue Disease; SLE: Systemic Lupus Erythematosus; RA: Rheumatoid Arthritis; SS:Sjogren's Syndrome.

-- indicates no diagnosis of concurrent rheumatic disease

Table 1. Demographics, Clinical Signs of Autonomic Dysfunction, Tilt Test Results and Concurrent Rheumatic Diseases of Patients Diagnosed with JHS

\begin{tabular}{|c|c|c|c|c|c|}
\hline Patient & Age & Gender & $\begin{array}{c}\text { Clinical } \\
\text { Signs of } \\
\text { Autonomic } \\
\text { Dysfunction }\end{array}$ & $\begin{array}{c}\text { Tilt Table } \\
\text { Test Results }\end{array}$ & $\begin{array}{c}\text { Concurrent } \\
\text { Rheumatologic } \\
\text { Diseases }\end{array}$ \\
\hline 1 & 22 & Female & + & + & $\mathrm{SpA}$ \\
\hline 2 & 39 & Female & + & + & -- \\
\hline 3 & 38 & Female & + & + & SLE \\
\hline 4 & 28 & Female & + & + & Behcet's \\
\hline 5 & 32 & Female & + & Not performed & UCTD \\
\hline 6 & 40 & Female & + & - & -- \\
\hline 7 & 37 & Female & + & + & SLE \\
\hline 8 & 28 & Female & + & + & Discoid Lupus \\
\hline 9 & 32 & Female & + & + & -- \\
\hline 10 & 48 & Female & + & - & SS \\
\hline 11 & 36 & Female & + & + & UCTD \\
\hline 12 & 32 & Female & + & + & -- \\
\hline 13 & 38 & Female & + & + & SS \\
\hline 14 & 39 & Female & + & + & UCTD \\
\hline 15 & 40 & Male & + & Not performed & -- \\
\hline 16 & 35 & Female & + & - & -- \\
\hline 17 & 35 & Female & + & - & RA \\
\hline 18 & 26 & Female & + & + & UCTD \\
\hline 19 & 33 & Female & + & Not performed & -- \\
\hline 20 & 22 & Female & + & + & SS \\
\hline 21 & 26 & Female & + & Not performed & SS \\
\hline 22 & 33 & Female & + & + & -- \\
\hline 23 & 55 & Female & + & + & SS \\
\hline 24 & 58 & Female & + & + & SS \\
\hline 25 & 28 & Female & + & + & -- \\
\hline 26 & 40 & Female & + & + & -- \\
\hline 27 & 44 & Female & + & + & UCTD \\
\hline 28 & 27 & Female & + & + & Behcet's \\
\hline 29 & 44 & Female & + & - & -- \\
\hline 30 & 31 & Female & + & Not performed & UCTD \\
\hline 31 & 18 & Female & + & Not performed & -- \\
\hline 32 & 31 & Female & + & + & -- \\
\hline 33 & 44 & Female & + & - & UCTD \\
\hline 34 & 30 & Male & + & - & -- \\
\hline 35 & 28 & Female & + & + & SLE \\
\hline 36 & 30 & Female & + & Not performed & SLE \\
\hline 37 & 40 & Male & + & Not performed & -- \\
\hline
\end{tabular}

increasing immunosuppression, despite attempts at advanced therapies in some patient cases. The patients were most commonly treated with increasing glucocorticoid therapy for presumed flare and at times, the patient's individualized immunosuppressive regimens were subject to increased dosing.

A systematic literature search was performed when we began to recognize a connection between our patients with joint hypermobility, fatigue, fibromyalgia-type symptoms and autonomic dysfunction. A detailed literature review of the PubMed database retrieved a few case reports of patients with hypermobility and chronic fatigue or fibromyalgia labels, which was more commonly found in adult Ehlers-Danlos Syndrome patients, whereas a few papers discussed the relationship between chronic fatigue and joint hypermobility in children. However, we were unable to find an extensive case series as noted in our facility for evaluating patients for joint hypermobility, 
autonomic dysfunction, and the associated and commonly reported poor quality of life in our patients.

We discovered that there was a selective group of patients with JHS who have autonomic dysfunction which contributes to the clinical manifestations with this creating a disorder in otherwise benign joint hypermobility situations. Thus, patients can often be inadvertently labeled at diagnoses with fibromyalgia, chronic fatigue or as having active connective tissue disease. These labels can interfere with treatment decisions. Potential manifestations of autonomic dysfunction can also occur which include cardiac dysrhythmias, postural orthostatic tachycardia syndrome, orthostatic hypotension and orthostatic intolerance. Although the underlying physiological mechanisms leading to such phenomena in JHS have not been clearly identified, they are likely to include reduced vascular tissue elasticity, and impaired peripheral vasoregulation as a consequence of adrenoreceptor or neuronal abnormalities [9].

An evaluation of JHS using the Brighton 1998 Criteria is a tool employed to help identify patients who may be falsely labeled as fibromyalgia and/or chronic fatigue and who may benefit from the interventions previously listed. Patients often fit criteria for multiple diagnoses and recognizing that a patient may coexist in multiple categories is necessary for adequate treatment. White et al. [10] reported on 74 patients labeled with fibromyalgia and found that $58 \%$ of the patients also met 1988 CDC criteria for the diagnosis of chronic fatigue syndrome. Branco et al. [11] reported on the prevalence of fibromyalgia in the general population compared to rheumatology outpatients using the London Fibromyalgia Epidemiological Study Screening Questionnaire (LFESSQ). In that study, 32\% of rheumatology outpatients screened positive for significant pain and fatigue compared to $13 \%$ in the general population. Fourteen percent of these rheumatology outpatients were labeled as fibromyalgia compared to $6.7 \%$ in the general population [11]. Accordingly, the US Centers for Disease Control reports of fibromyalgia coexisting 25-65\% of the time in connection with other connective tissue diseases have been noted [12]. As discussed by Ahn and Goldman-Ramsey [13], there remain limitations in measurements of fatigue in SLE whereby research showed fatigue experienced in $53-80 \%$ of lupus patients and the pain/fatigue combination experienced in $95 \%$ of lupus patients. Given the significant impact of HyPOTS on the quality of life, these patients should be screened for hypermobility accordingly.

The diagnosis of HyPOTS is recognized as leading to increased treatment options for patients suffering from the aforementioned manifestations of this disorder. This would involve, a well-rounded approach to managing postural tachycardia and include adequate salt and water intake, compression stockings, mineralocorticoid administration, along with physiotherapy techniques, consisting of joint and core muscle strengthening, orthotics, which taken together can improve these patients symptoms and quality of life [7]. In fact, the main point to be taken away from this retrospective analysis is that many of the clinical symptoms attributed to primary connective tissue disease could, in fact, be related to joint hypermobility. Once made aware of this problem from which a patient's autonomic disturbance is confirmed by a positive tilt table test, future instructions to the patient start with improving support of the lower extremities through the use of compression stockings and adequate salt and water intake. Providing the patient with an overall awareness of the problem empowers the patient to take control of the symptoms.

The patients in our study continue to be followed within our rheumatology division for symptomatic management of HyPOTS, often in conjunction with a team of specialists, including those practicing cardiology and neurology. We have found that treatment of HyPOTS using the interventions indicated above have led to significant improvement in the quality of life of these patients as we continue to recognize new cases of patients with HyPOTS.

\section{Significance and innovations}

- Joint hypermobility syndrome is a chronic disorder which presents with arthralgia, myalgia and at times in association with visceral manifestations and POTS (postural orthostatic tachycardia syndrome).

- Patients with joint hypermobility syndrome are more likely to be diagnosed with chronic fatigue syndrome or fibromyalgia compared to the general population.

- HyPOTS (joint hypermobility syndrome associated with postural orthostatic tachycardia syndrome) has been underrepresented in rheumatologic literature. Clinical findings of HyPOTS may be considered to be complications of patient's underlying connective tissue disease, resulting in increased treatment of the connective tissue disease.

Interventions, such as maintaining adequate hydration along with use of compression stockings, rather than intensifying the treatment of an underlying connective tissue disease, lead to improvements in symptoms.

\section{Acknowledgements}

An abstract of this study which was co-authored by Artan Kaso, M.D. and Ali D. Askari, M.D was presented at the 2014 American College of Rheumatology Annual Meeting.

\section{Conflict of interest}

None to be declared.

\section{References}

1. Hakim A, Grahame R (2003) Joint hypermobility. Best Pract Res Clin Rheumatol 17 989-1004. [Crossref]

2. Folci M, Capsoni F (2016) Arthralgias, fatigue, paresthesias and visceral pain: can joint hypermobility solve the puzzle? A case report. BMC Musculoskelet Disord 17: 58. [Crossref]

3. Gazit Y, Nahir AM, Grahame R, Jacob G (2003) Dysautonomia in the joint hypermobility syndrome. Am J Med 115: 33-40. [Crossref]

4. Haensch C, Tosch M, Katona I, Weis J, Isenmann S (2014) Small-fiber neuropathy with cardiac denervation in postural tachycardia syndrome. Muscle Nerve 50: 956-961. [Crossref]

5. De Wandele I, Calders P, Peersman W, Rimbaut S, De Backer T, et al. (2014) Autonomic symptom burden in the hypermobility type of Ehlers-Danlos syndrome: A comparative study with two other EDS types, fibromyalgia, and healthy controls. Semin Arthritis Rheum 44: 353-361. [Crossref]

6. Barron DF, Cohen BA, Geraghty MT, Violand R, Rowe PC (2002) Joint hypermobility is more common in children with chronic fatigue syndrome than in healthy controls. $J$ Pediatr 141: 421-425. [Crossref]

7. Grahame R, Bird HA, Child A (2000) The revised (Brighton 1998) criteria for the diagnosis of benign joint hypermobility syndrome (BJHS). J Rheumatol 27: 17771779. [Crossref]

8. Benarroch EE (2012) Postural tachycardia syndrome: a heterogeneous and multifactorial disorder. Mayo Clin Proc 87: 1214-1225. [Crossref]

9. Bonyhay I, Freeman R (2004) Sympathetic nerve activity in response to hypotensive stress in the postural tachycardia syndrome. Circulation 110: 3193-3198. [Crossref] 
10. White KP, Speechley M, Harth M, Ostbye T (2000) Co-existence of chronic fatigue syndrome with fibromyalgia syndrome in the general population. A controlled study. Scand J Rheumatol 29: 44-51. [Crossref]

11. Branco JC, Bannwarth B, Failde I, Abello Carbonell J, Blotman F, et al. (2010) Prevalence of fibromyalgia: a survey in five European countries. Semin Arthritis Rheum 39: 448-453. [Crossref]
12. Fibromyalgia | Arthritis | CDC. Cdcgov. 2016. Available at: http://www.cdc.gov/arthritis/basics/fibromyalgia.htm\# Accessed August 3, 2016.

13. Ahn GE, Ramsey-Goldman R (2012) Fatigue in systemic lupus erythematosus. Int $J$ Clin Rheumtol 7: 217-227. [Crossref]

Copyright: (C2017 Mandel D. This is an open-access article distributed under the terms of the Creative Commons Attribution License, which permits unrestricted use, distribution, and reproduction in any medium, provided the original author and source are credited. 